\title{
Characterization of Porous GaN-Nanostructured Rods by TEM Techniques
}

\author{
Jian-Guo Zheng*, Sha Ding ${ }^{+}$, Mingmei $\mathrm{Wu}^{+}$
}

*NUANCE center and Department of Materials Science \& Engineering, Northwestern University, Evanston, IL 60208, USA

${ }^{+}$State Key Laboratory of Optoelectronic Materials and Technologies, School of Chemistry and Chemical Engineering, Sun Yat-Sen (Zhongshan) University, Guangzhou, 510275, People's Republic of China

$\mathrm{GaN}$ is a wide bandgap semiconductor and has been a subject of intensive research for $\mathrm{UV}$ and blue light emission, high temperature electronic devices and chemical gas sensor applications. It is important to control the growth of the material because of its anisotropic properties. Significant progress was made recently by Yang and co-workers who successfully prepared single crystal GaN nanotubes using a $\mathrm{ZnO}$ template and $\mathrm{CVD}$ method [1]. Here we report porous $\mathrm{GaN}$ rods composed of well textured nanoparticles. These GaN nanostructured rods were prepared by a facile chemical method without a template and characterized mainly by various TEM techniques.

Fig. 1 shows a bright field TEM image of some broken GaN rods with a typical diameter of 200-400 $\mathrm{nm}$. The rod length may range from several microns to 20 microns. EDX spectrum (Fig. 2) indicates that these rods consist of $\mathrm{Ga}$ and $\mathrm{N}$, which was further confirmed by EELS spectroscopy (inset of Fig. 2). Small amount of oxygen revealed from the EELS spectrum is attributed to surface oxidation. $\mathrm{X}$-ray diffraction and selected area electron diffraction show that the GaN rods have a wurtzite crystal structure which is consistent with PDF\# 50-0792 ( $a=3.18907 \AA$, and $c=5.1855 \AA)$.

The GaN rods exhibit three apparent characteristics. First, they contain a lot of voids, which can be readily seen from Fig. 1. This is further confirmed by high angle annual dark field (HAADF) STEM image (Fig. 3), EELS thickness map and EDX line scan (inset of Fig.3). The porous rods may be a very good candidate for gas sensor application at high temperature because of high melting point of $\mathrm{GaN}$. Second, the GaN rods are composed of nanoscale particles with a diameter of 20-30 nm. 3D morphology of the nanoparticles may be clearly seen from SEM images. Dark field TEM image provides further evidence about the nature of nanostructured rods (Fig. 4). Third, these nanoparticles are well textured (Fig. 5), that is, the [11 20$]$ direction of the nanoparticles is parallel or nearly parallel to the axis of the GaN rods (Fig. 6). We anticipate that the properties of these rods should be different from normal polycrystalline GaN rods.

In conclusion, we have successfully prepared porous nanostructured and well-textured $\mathrm{GaN}$ rods. Investigations on their structure-related properties are in progress.

[1] J. Goldberger et al., Nature, 422 (2003), 599.

[2] The TEM work was performed in the EPIC facility of NUANCE Center at Northwestern University. NUANCE Center is supported by NSF-NSEC, NSF-MRSEC, the State of Illinois, and Northwestern University. The samples were synthesized in the State Key Laboratory of Optoelectronic Materials and Technologies, School of Chemistry and Chemical Engineering, Sun Yat-Sen (Zhongshan) University, China, and supported by the government of Guangdong Province and NSF of China. 


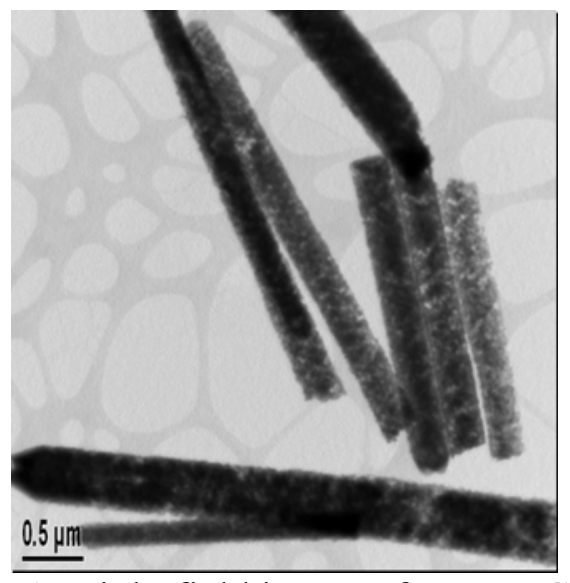

Fig. 1 Bright field image of porous GaN rods

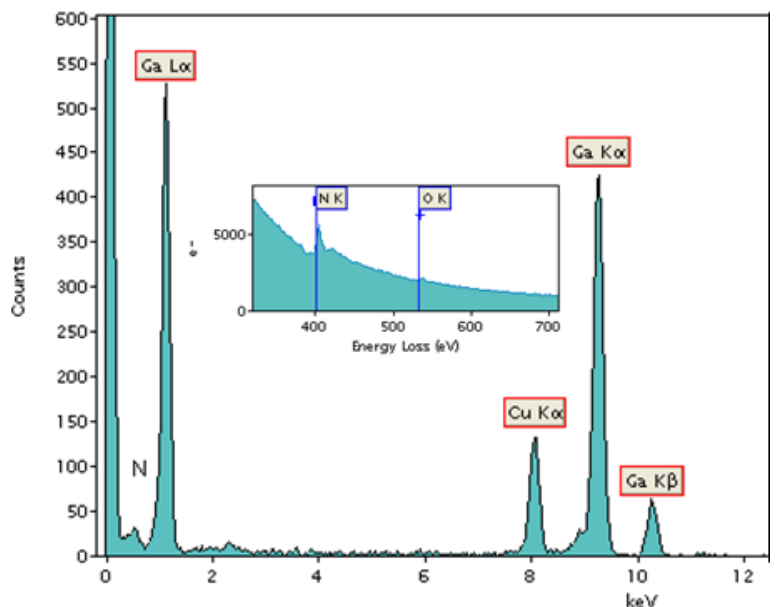

Fig. 2 EDX spectrum and EELS spectrum (inset) of $\mathrm{GaN}$ rod. $\mathrm{Cu}$ signal comes from supporting grid.

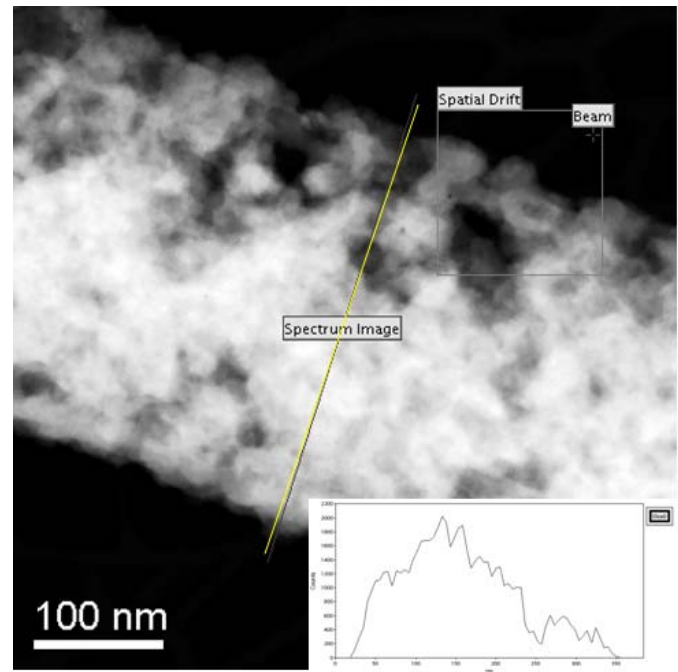

Fig. 3 HAADF STEM image of porous GaN rod and Ga profile across the rod (inset).

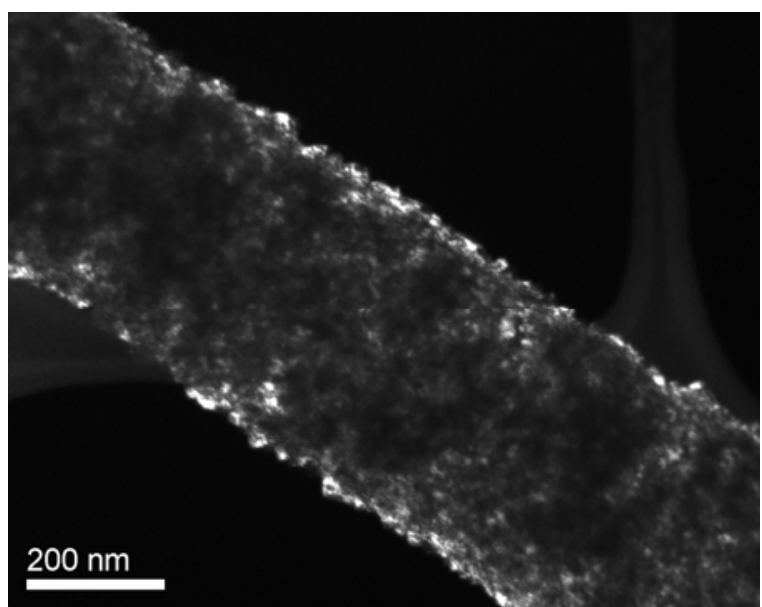

Fig. 4 GaN nanoparticles in $\mathrm{GaN}$ rod

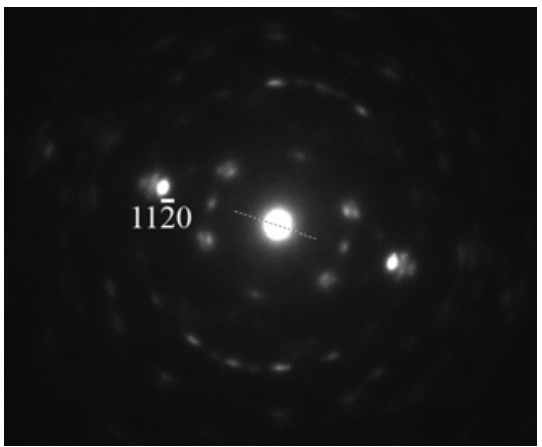

Fig. 5 Selected area diffraction pattern of GaN rod showing strong texture. The dashed line across the transmission spot is parallel to the rod axis.

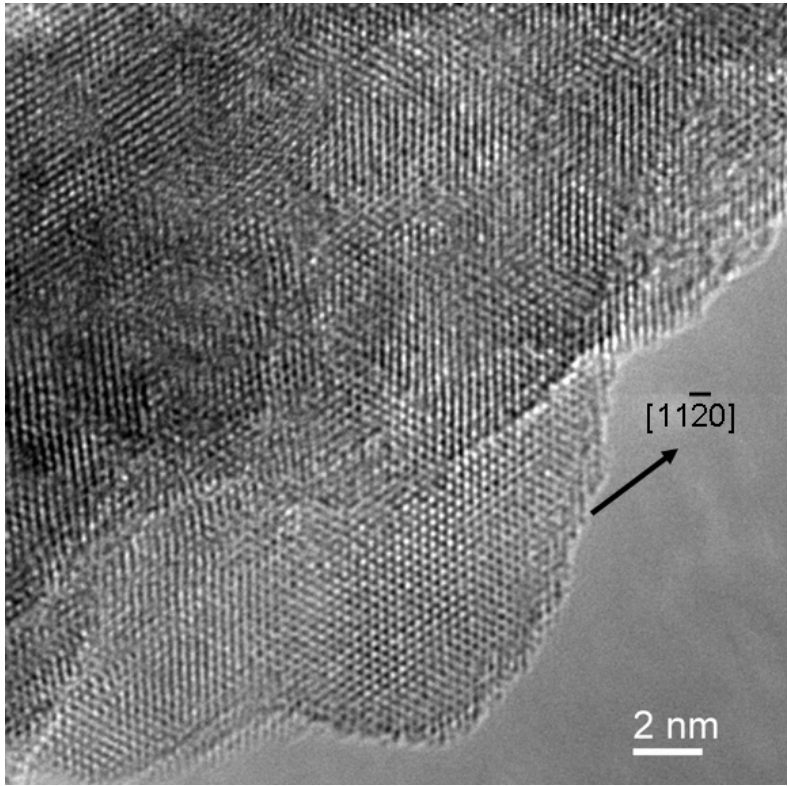

Fig. 6 HRTEM image of GaN nanoparticles. 